\title{
Experimental investigation of random noise-induced beam degradation in high-intensity accelerators using a linear Paul trap
}

\author{
Moses Chung \\ Accelerator Physics Center, Fermi National Accelerator Laboratory, Batavia, Illinois 60510, USA
}

Erik P. Gilson, Ronald C. Davidson, Philip C. Efthimion, and Richard Majeski

Plasma Physics Laboratory, Princeton University, Princeton, New Jersey 08543, USA

(Received 3 February 2009; published 26 May 2009)

\begin{abstract}
A random noise-induced beam degradation that could affect intense beam transport over long propagation distances has been experimentally investigated by making use of the transverse beam dynamics equivalence between an alternating-gradient focusing system and a linear Paul trap system. For the present study, machine imperfections in the quadrupole focusing lattice are considered, which are emulated by adding small random noise on the voltage waveform of the quadrupole electrodes in the Paul trap. It is observed that externally driven noise continuously increases the rms radius, transverse emittance, and nonthermal tail of the trapped charge bunch almost linearly with the duration of the noise. The combined effects of collective modes and colored noise are also investigated and compared with numerical simulations.
\end{abstract}

DOI: 10.1103/PhysRevSTAB.12.054203

PACS numbers: 52.59.Sa, 29.27.- a, 41.85.Ja, 52.27.Jt

\section{INTRODUCTION}

High-intensity accelerators have a wide range of applications ranging from basic scientific research in high energy and nuclear physics, to applications such as heavy ion fusion, ion-beam-driven high energy density physics, tritium production, nuclear waste transmutation, and spallation neutron sources for material and biological research [1-3]. One critical but unavoidable problem in highintensity accelerators is the presence of undesired random noise due to machine imperfections, and its influence on the long-time-scale beam dynamics [4,5]. The machine imperfections may include quadrupole magnet and rf cavity alignment errors, quadrupole focusing gradient errors, rf field amplitude and phase errors, to mention a few examples [6]. Usually, random noise in the machine components acts as a continuous supply of free energy to the beam, which results in irregular mismatch oscillations of the beam envelope [5], enhanced halo formation [4,7], and emittance growth [8], particularly over long propagation distances. Consequently, the associated beam degradation defines the practical and/or economic tolerances in the machine design and operation [3,9]. In transforming random noise effects into emittance growth, the action of the nonlinear space-charge force plays a critical role [8]. Hence, it is increasingly important to understand the effects of random noise on long-distance beam propagation with moderate space-charge forces. From various multiparticle simulations with both space-charge and random noise effects, considerable progress has been made in developing an improved understanding of the random noise-induced beam degradation [5,6,8,10-12]. However, experimental verification of these effects has been some- what limited due to the lack of dedicated experimental facilities that allow the study of long-time-scale phenomena.

The Paul trap simulator experiment (PTSX), which is a linear Paul trap [13] that can experimentally simulate the nonlinear transverse dynamics of intense beam propagation over large equivalent distances through an alternatinggradient (AG) transport lattice [14,15], provides a compact and flexible laboratory facility for the experimental investigation of random noise effects. The idea of using a linear Paul trap confining a pure ion plasma to study intense beam propagation was proposed by Davidson et al. [14] and by Okamoto and Tanaka [16]. The physics equivalence between the AG system and the linear Paul trap system, including the self-field forces, is described in detail in Ref. [14]. The PTSX device consists of three collinear cylinders with radius $r_{w}=0.1 \mathrm{~m}$, each divided into four $90^{\circ}$ azimuthal sectors (see Fig. 1 in Sec. II for details). The pure ion plasma is confined transversely in the central $2 \mathrm{~m}$ long cylinder by oscillating voltages. The static voltages on the two $0.4 \mathrm{~m}$-long end cylinders confine the ions axially. A brief description of the PTSX device is given in Sec. II of this paper. The amplitude of the oscillating voltage waveform applied to the central electrodes in the PTSX device corresponds to the quadrupole focusing gradient in an AG lattice system. Hence, by slightly modifying the voltage amplitude $V_{0 \max }$ in every half-focusing period $T / 2$, we can simulate the effect of randomly distributed quadrupole focusing gradient errors in the actual transport channel.

As mentioned earlier, in intense beams, the action of the nonlinear space-charge force plays a critical role in transforming random noise effects into emittance growth [8]. The relative importance of space-charge effects can be 
(a)

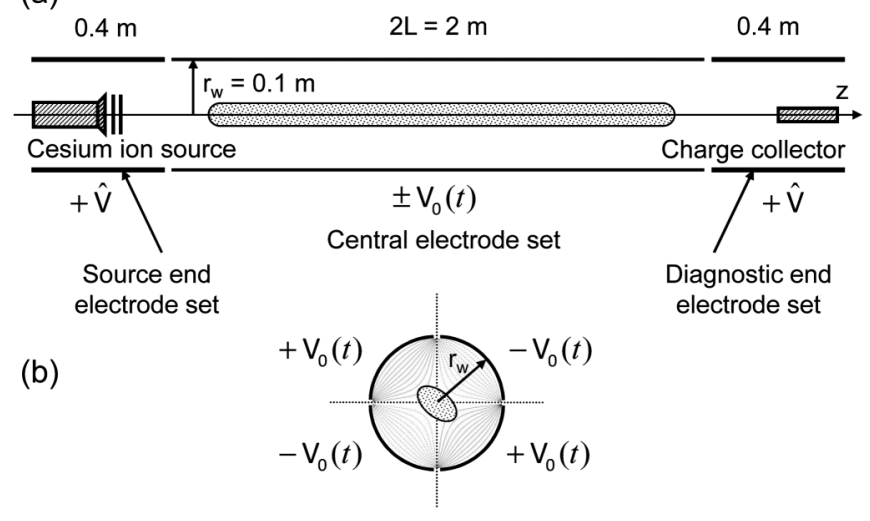

FIG. 1. Schematic of the PTSX device showing: (a) side view of the quadrupole electrodes, cesium ion source, and charge collector, and (b) end view of the central electrode set.

described either in terms of the effective tune depression $\nu / \nu_{0}$ [17], or the normalized intensity parameter $\hat{s}=$ $\omega_{p}^{2}(0) / 2 \omega_{q}^{2}$ [18], where $\omega_{p}^{2}(r)=n(r) q^{2} / m \epsilon_{0}$ is the plasma frequency squared, and $\omega_{q}$ is the average smooth-focusing frequency of the beam particles' transverse oscillations in the applied focusing field. Here, $\epsilon_{0}$ is the permittivity of free space, $n(r)$ is the radial ion density profile, $r$ is the radial distance from the beam axis, and $q$ and $m$ are the ion charge and mass, respectively. For example, the newly commissioned Spallation Neutron Source (SNS) is designed to be operated at $\nu / \nu_{0} \geqslant 0.6$ in the linac section (total length $\sim 331 \mathrm{~m}$ ), and $\nu / \nu_{0} \gtrsim 0.9$ in the accumulator ring (circumference $\sim 248 \mathrm{~m}$ ) $[19,20]$, which correspond to $\hat{s} \lesssim 0.9$ and $\hat{s} \lesssim 0.35$, respectively. In the linac section, space-charge effects play a more important role for the noise effect to be significant. In the accumulator ring, on the other hand, the long propagation distances associated with the beam lifetime can have a larger impact on noiseinduced beam degradation. Since the PTSX device covers the operating range of $0<\hat{s}<0.8$, and can confine the charge bunch up to $300 \mathrm{~ms}$, which is equivalent to nearly $20 \mathrm{~km}$-long beam propagation distances [15] for an equivalent AG system with 1-m magnet spacing, effects of the quadrupole focusing gradient errors in SNS-like highintensity accelerators can be effectively studied. The typical error limits of the quadrupole focusing gradients in the various components of SNS are given in Table I.

TABLE I. Values of quadrupole gradient error limits for various components of the SNS linac and accumulator ring [20,21].

\begin{tabular}{lc}
\hline \hline Component & Limit on error \\
\hline Medium energy beam transport (MEBT) & $1.732 \%$ \\
Drift tube linac (DTL) & $0.5 \%$ \\
Coupled-cavity drift tube linac (CCDTL) & $0.25 \%$ \\
Coupled-cavity linac (CCL) & $0.25 \%$ \\
Accumulator ring & $0.01 \%$ \\
\hline \hline
\end{tabular}

In performing the actual experiments on noise effects in PTSX, however, it is important to minimize any other sources of beam state change that might be comparable to the random noise effect, such as injection beam mismatch, two-stream interactions, collision with background neutral gas, or a drift in the ion source conditions. Hence, for the initial experiments presented in this study, we use a moderately low space-charge-density beam $(\hat{s} \sim 0.2$, or equivalently, effective tune depression [17] $\nu / \nu_{0} \sim 0.95$ ) that has been carefully optimized through the injection scheme described in Ref. [22]. The results of this study are more applicable to typical high-intensity rings where $\nu / \nu_{0}=0.9-0.96$ rather than to space-charge dominated machines such as heavy ion linacs with $\nu / \nu_{0}<0.5$ [23]. The optimized plasma is held in a quasiequilibrium state for about $38 \mathrm{~ms}$ (see Fig. 3), which is equivalent to the quiescent beam propagation over about 2280 full AG focusing periods for the focusing frequency of $f_{0}=$ $60 \mathrm{kHz}$. Here, the transverse defocusing space-charge force is about $10 \%$ of the applied transverse focusing force, and other possible sources of thermalization of the free energy that may lead to emittance growth in the absence of the space charge, such as collisions with the background gas and nonlinearities in the applied focusing force [14], are estimated to be negligibly small for the present experimental conditions.

In addition, the number of error samples (independent set of time series of random numbers used for modifying the focusing field strength) is also an important factor for obtaining good statistics in the experimental data [10]. In multiparticle simulations, the number of error samples has been chosen as small as 20 [11], or as large as 500 [5], depending on the computation time and required accuracy for the quantitative analysis. For the experimental studies reported in this study, we use 20 error samples for a given error limit and trapping time (which is equivalent to a given propagation distance in the actual AG lattice). In this way, we can reduce the overall experimental time, avoiding any possible drift in the experimental conditions (mostly in the ion source) during the course of scanning the entire parameter range. A description of the PTSX facility including the cesium ion source and radially scanning charge collector is given in Sec. II, and a smooth-focusing model for the analysis of the noise-induced beam degradation is described in Sec. III. Experimental results with uniform white noise are presented in Sec. IV, and the effects of colored noise are discussed in Sec. V.

\section{EXPERIMENTAL APPARATUS}

The Paul trap simulator experiment (PTSX) device is a linear Paul trap [13,15] constructed from a $2.8 \mathrm{~m}$-long, $r_{w}=10 \mathrm{~cm}$-radius, gold-plated stainless steel cylinder (Fig. 1). The cylinder is divided into two $40 \mathrm{~cm}$-long end cylinders and a $2 L=2 \mathrm{~m}$-long central cylinder. All cylinders are azimuthally divided into four $90^{\circ}$ sectors so that 
when an oscillating voltage $V_{0}(t)$ is applied with alternating polarity on adjacent segments, the resulting electric field becomes an oscillating quadrupole field near the trap axis. This quadrupole electric field exerts a ponderomotive force that confines the pure ion plasma radially. To trap the plasma axially, the two end electrodes are biased to a constant positive voltage $+\hat{V}$, after the charge bunch is injected into the central section.

The cesium ion source is located on the trap axis near the center of one of the short electrode sets so that ion injection is not affected by the fringe fields [24]. The amount of charge injected can be controlled easily by adjusting the voltages on the emitter surface $\left(V_{s}\right)$, acceleration grid $\left(V_{a}\right)$, and deceleration grid $\left(V_{d}\right)$ of the ion source. A DC power supply keeps the source temperature around $1000^{\circ} \mathrm{C}$ so that cesium is ionized through contact ionization on the emitter surface. The charge collector is mounted on a linear motion feedthrough at the other end of the short electrode set, and moves in the transverse direction along a null of the applied potential in order to minimize the perturbation of the quadrupole potential configuration during the profile measurement [25]. Using the sensitive electrometer with LABVIEW interface, the total axially integrated ion charge $Q(r)$ through the collector plate centered at radius $r$ can be measured accurately to as low as the $1 \mathrm{fC}$ range, which is adequate to detect the formation of halo particles. The radial ion density profile $n(r)$ is related to $Q(r)$ by $n(r) \approx$ $Q(r) / q \pi r_{c}^{2} L_{p}$. Here, $r_{c}$ is the size of the circular collecting plate and $L_{p}$ is the plasma length. The effect of finite width of the collector plate has been corrected during the data processing stage, and the resultant error is only a few percent.

The PTSX device manipulates the charge bunch using an inject-trap-dump-rest cycle, and the one-component plasmas created in the trap are highly reproducible [15]. A sinusoidal waveform $V_{0}(t)=V_{0 \max } \sin \left(2 \pi f_{0} t\right)$ is applied by an arbitrary function generator with a $20 \mathrm{MHz}$ clock rate. Random noise is excited through another LABVIEW interface which samples a uniformly distributed random number $\delta$ in the range $|\delta| \leq \Delta_{\max }$, and adjusts $V_{0 \max }$ to $V_{0 \max }(1+\delta)$ in every half-focusing period $1 / 2 f_{0}$. Application of the noise signal begins after the injected ion bunch becomes sufficiently stabilized (typically in about $12 \mathrm{~ms}$ ), and ends before the dumping stage begins. In the dumping stage, most of the trapped ions are collected within $2 \mathrm{~ms}$ without significant changes in the radial density profile. Since the collected charge is necessarily averaged over many focusing periods during the dumping process, the value of the rms radius calculated from the measured radial profile can be interpreted as the rms radius of the beam in the smooth-focusing approximation [18].

In order to minimize the effects of neutral collisions on the plasma behavior, the base pressure of PTSX is kept below $5 \times 10^{-9}$ Torr after a week-long bake at $200^{\circ} \mathrm{C}$.
When the ion source is on, the operating pressure rises up to $10^{-8} \sim 10^{-7}$ Torr. Even in this case, the characteristic ion-neutral collision time is $\tau_{\text {in }} \gtrsim 2 \mathrm{sec}$, and the trapped plasma is collisionless to very good approximation. The vacuum phase advance used in this experiment is $\sigma_{v}=52^{\circ}$, which is considerably below the envelope instability limit [18]. When $\sigma_{v}<72^{\circ}$, it is well established that the smooth-focusing model, in which the average beam cross section is assumed to be circular, is a good approximate description of a beam with elliptical cross section in an AG lattice [18]. Note also that recent axial current measurements [26] show that the axial nonuniformity in the initial beam is smoothed out in about $6 \mathrm{~ms}$.

\section{SMOOTH-FOCUSING THEORETICAL MODEL}

For simplicity in the theoretical analysis presented in this section, we assume that the average effects of the quadrupole focusing field are described by an equivalent smooth-focusing force [18]. In this smooth-focusing approximation, the evolution of the rms radius $R_{b}(t)$ of a charge bunch with line density $N$ is effectively described by

$$
\frac{d^{2} R_{b}}{d t^{2}}+\omega_{q}^{2} R_{b}-\frac{K}{2 R_{b}}-\frac{\epsilon^{2}}{4 R_{b}^{3}}=0
$$

where $\omega_{q}$ is the smooth-focusing frequency in the absence of noise, $K=2 N q^{2} / 4 \pi \epsilon_{0} m$ is the effective (dimensional) self-field perveance, and $\epsilon=2 R_{b}\left[\left\langle\dot{x}^{2}+\right.\right.$ $\left.\left.\dot{y}^{2}\right\rangle_{f}-\left(d R_{b} / d t\right)^{2}\right]^{1 / 2}$ is the average transverse emittance defined in the beam frame [18]. Here, $\langle\cdots\rangle_{f}$ denotes the statistical average over the particle distribution function in the smooth-focusing approximation. Note that the effective self-field perveance $K$ and the average transverse emittance $\epsilon$ have the dimensions of (velocity) ${ }^{2}$ and (length $\times$ velocity), respectively. For a sinusoidal voltage waveform $V_{0}(t)=V_{0 \max } \sin \left(2 \pi f_{0} t\right)$ used in the PTSX, the smoothfocusing frequency occurring in Eq. (1) is given approximately by [18]

$$
\omega_{q}=\frac{8 q V_{0 \max }}{m \pi r_{w}^{2} f_{0}} \frac{1}{2 \sqrt{2} \pi} .
$$

When there are random errors in voltage amplitudes $V_{0 \text { max }}$ in every half-focusing period $T / 2=1 / 2 f_{0}$, the trapped plasma encounters a time series in the smooth-focusing frequencies given by $\omega_{q}\left(1+\delta_{1}\right), \ldots, \omega_{q}\left(1+\delta_{i}\right)$, $\ldots, \omega_{q}\left(1+\delta_{N_{1 / 2}}\right)$ [8]. Here, $\delta_{i}$ is a random number which belongs to a uniform distribution in the range $-\Delta_{\max } \leq$ $\delta_{i} \leq \Delta_{\max }$, and $i$ and $N_{1 / 2}$ are the index and the total number of half periods of the noise duration, respectively. Hence, an error sample is composed of $N_{1 / 2}$ statistically independent random numbers, representing white noise in the present analysis. 
An example of the numerical solution of Eq. (1), having an error sample with $\Delta_{\max }=1 \%$, shows that irregular oscillations of the beam envelopes are excited in a manner similar to beam mismatch [5] and grow continuously to considerable amplitudes, depending on the duration of the noise [Fig. 2(a)]. Since the envelope equation itself cannot self-consistently describe the emittance growth resulting from the envelope oscillations, the center of the oscillations remains nearly the same as the initial equilibrium radius $R_{b 0}$. To describe the time evolution of the transverse emittance self-consistently, we make use of particle-in-cell (PIC) simulations. The result of WARP 2D PIC simulations [27] with the same error sample used in the envelope equation demonstrates that the oscillation amplitudes are saturated to some extent, and that the oscillation center increases linearly with the noise duration [Fig. 2(b)], implying the conversion of free energy available from the envelope oscillations into emittance growth [28]. Even though there are as many negative energy kicks as positive kicks on the average, the simulation results suggest that the random noise has a cumulative effect [5], which ends up
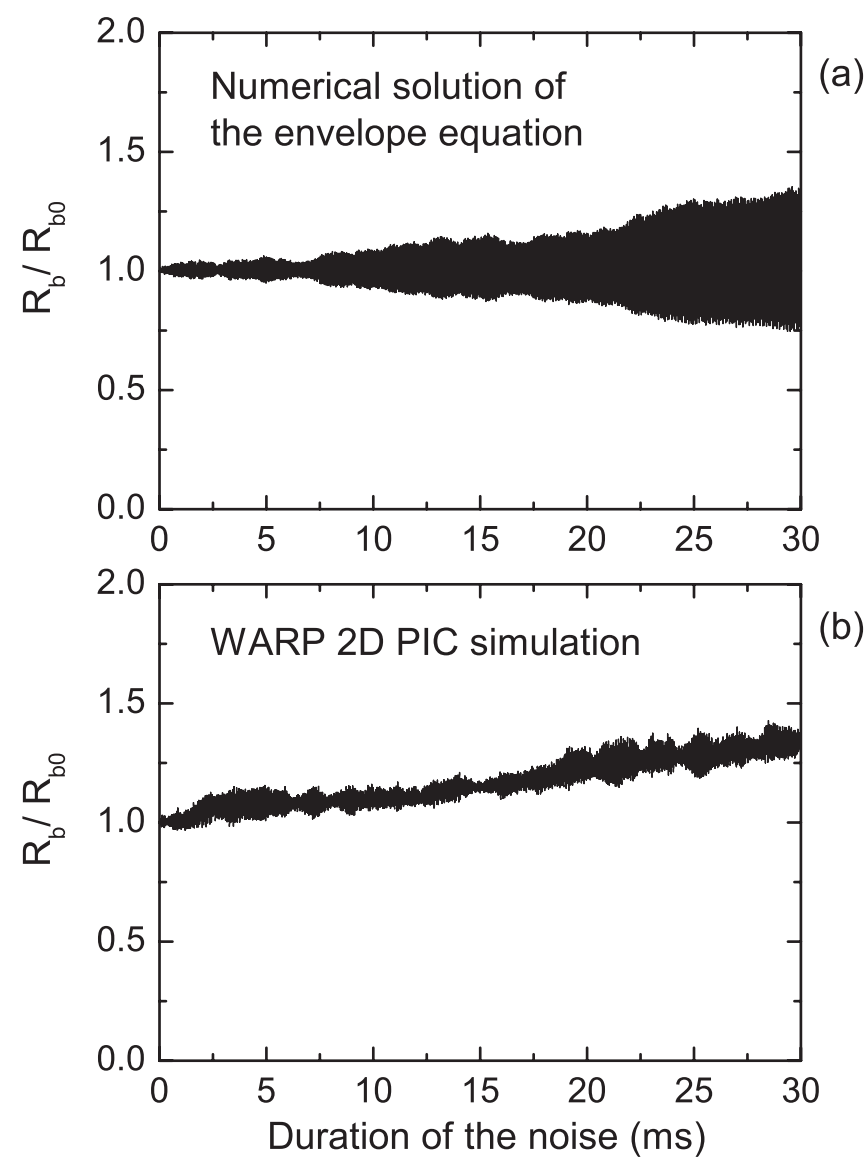

FIG. 2. Evolution of the rms radius $R_{b}(t)$ calculated (a) from the envelope equation with constant emittance, and (b) from 2D WARP simulations with emittance growth. Note that the same error sample with $\Delta_{\max }=1 \%$ is used for (a) and (b). producing an overall increase in transverse energy (temperature) of the beam particles.

Since the error limit $\Delta_{\max }$ in the focusing force is typically only a few percent, any possible changes due to the random noise in the global quantities such as the line density $N$, the effective transverse temperature $\bar{T}_{\perp}$, and the average transverse emittance $\epsilon$, will take place quite slowly. In this case, we can still assume that the beam is in a quasiequilibrium state, which means that the average focusing force balances both the thermal pressure force of the plasma and the space-charge force over a slow time scale. Therefore, the global force balance equation can be expressed approximately as [18]

$$
m \omega_{q}^{2} R_{b}^{2}=2 \bar{T}_{\perp}+\frac{N q^{2}}{4 \pi \epsilon_{0}}
$$

and the evolution of the average transverse emittance can be approximated by

$$
\epsilon(t)=2 R_{b}\left(\omega_{q}^{2} R_{b}^{2}-\frac{N q^{2}}{4 \pi \epsilon_{0} m}\right)^{1 / 2}
$$

Indeed, Eqs. (3) and (4) are equivalent in form to the envelope equation (1), provided we set $d^{2} R_{b} / d t^{2}=0$ in Eq. (1) (equilibrium case). Equation (4) allows us to estimate the emittance growth due to the random noise, simply by using the values of $N$ and $R_{b}$ measured in the experiments. Note that errors in calculating the emittance using Eq. (4) result primarily from the instrumental uncertainties at large radii (see error bars in Fig. 6). However, when there exists a significant population of low-density halo particles below the detection limit ( $\lesssim 1 \mathrm{fC}$ ) of the charge collector, then the emittance calculated from Eq. (4) necessarily underestimates the actual mean transverse emittance. Particles far away from the beam core $\left(>\sqrt{2} R_{b}\right)$ are of course weighted more heavily in calculating the emittance in the simulations [12,27].

\section{EXPERIMENT RESULTS}

The initial plasma is obtained $12 \mathrm{~ms}$ after the ion injection is completed. Figure 3 shows that the measured radial charge profile $Q(r)$ is approximately a Gaussian function of $r$ (a straight line in the log versus $r^{2}$ plot as low as to the $1 \mathrm{fC}$ range) with a temperature comparable to the thermal temperature of the cesium ion source $\left(\sim 1000^{\circ} \mathrm{C}\right)$, indicating the validity of the assumption of thermal-equilibrium-like beam.

To characterize the statistical properties of the beam response to the random noise, we make use of the onaxis charge $\hat{Q}=Q(r=0)$, which is the most easily and accurately measurable quantity in the present experimental setup. Figure 4 shows the time history of the statistical average $(\langle\hat{Q}\rangle)$, and the standard deviation (square root of 


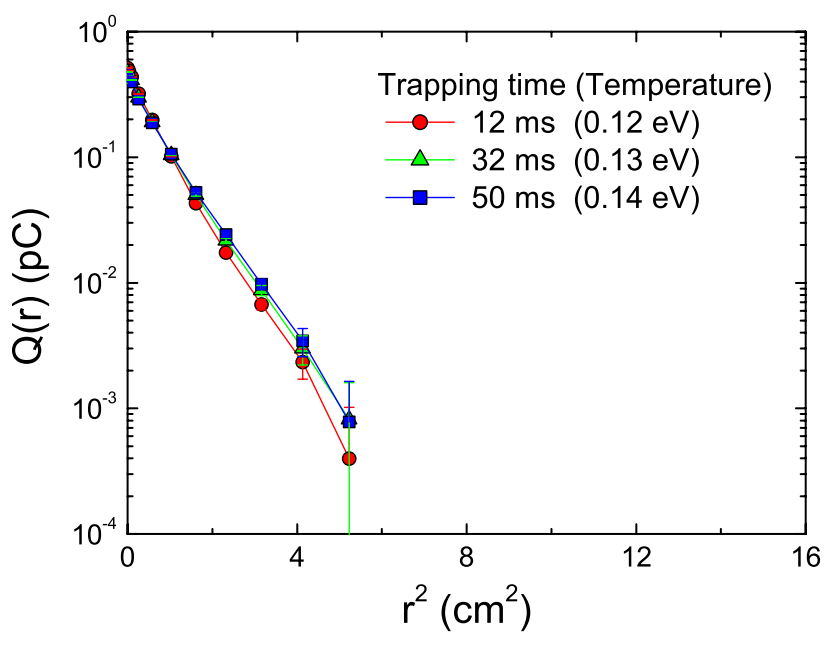

FIG. 3. (Color) Measured radial profiles of trapped plasma without applied noise. The charge bunch is maintained in the quasiequilibrium state for about $38 \mathrm{~ms}$ with a slight increase in the effective transverse temperature [calculated from Eq. (3)]. A straight line in the log of $Q(r)$ versus $r^{2}$ plot indicates that the radial profile is a Gaussian function of $r$.

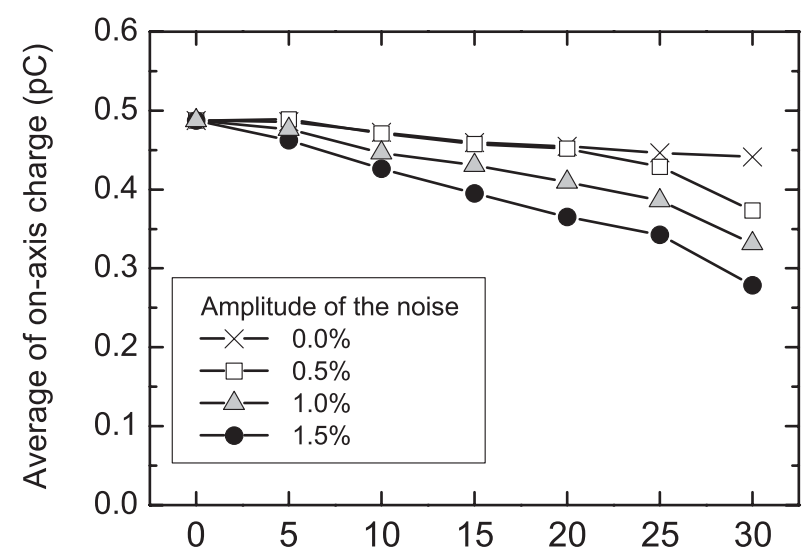

(a)

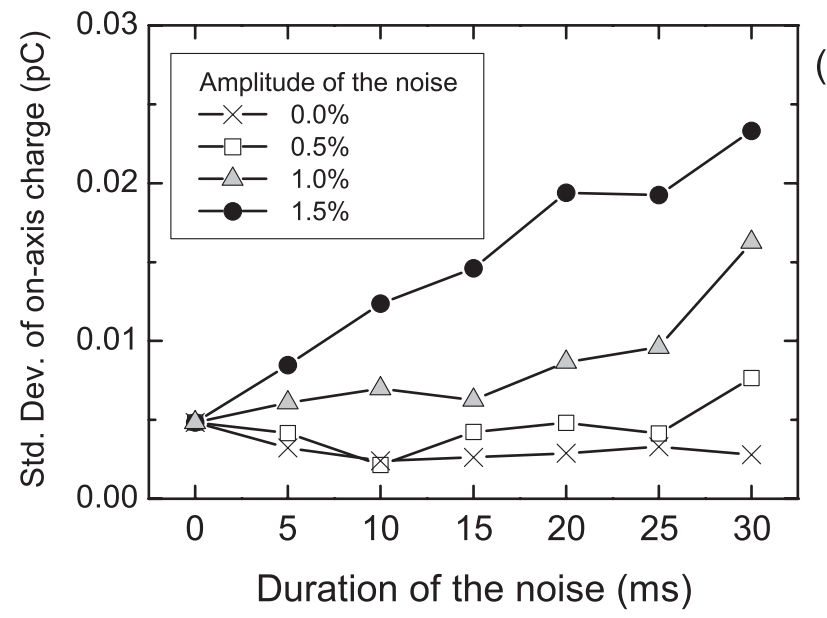

FIG. 4. Time history of (a) the statistical average, and (b) the standard deviation of the on-axis charge computed over an ensemble of 20 random error samples. the variance $\sigma_{\hat{Q}}^{2}=\left\langle\hat{Q}^{2}\right\rangle-\langle\hat{Q}\rangle^{2}$ ) of the on-axis charge computed over an ensemble of 20 random error samples. The average of the on-axis charge decays almost linearly with the amplitude and the duration of the noise up to $25 \mathrm{~ms}$. After $25 \mathrm{~ms}$, the decay rate becomes somewhat rapid, which is likely related to the production of halo particles [see also Figs. 5(a) and 7] and the resultant enhanced particle loss. It should be noted that both beam expansion due to emittance growth and halo production due to parametric resonance are responsible for the on-axis charge decay observed in this experiment. As illustrated in Fig. 2, emittance growth leads to beam expansion, which is necessarily accompanied by core depletion when the line density $N$ is fixed. In addition, a particle-core model [5] predicts that noise-induced mismatch oscillations can also populate low-density halo particles at large radii, particularly after long propagation distances (trapping time in this experiment). Detailed numerical studies (similar to Ref. [5]) may help to understand more clearly the role of such mechanisms on core depletion. Because the initial beam state is not a perfect equilibrium, and there is intrinsic noise (either physics originated, or device originated) in the PTSX device, such as two-stream interactions, collisions with residual gas, drift in the ion source conditions, jitter in the voltage waveform, or mechanical vibrations of the vacuum pumps, the on-axis charge for the case with no applied noise also decreases slightly during the $30 \mathrm{~ms}$ of trapping. The fact that this process has a nearly constant standard deviation suggests that instrumental uncertainties are dominant over small-amplitude intrinsic noise. Because of the relatively small number of error samples, it is not clear if the beam response is a random-walk-like diffusion process $\left(\sigma_{\hat{Q}} \propto t^{1 / 2}\right)$ [29]. However, the general tendency is that the standard deviation of the on-axis charge increases with time, which strongly suggests that the fluctuations in the on-axis charge measurements originate from the applied noise rather than from instrumental uncertainties (i.e., $\sigma_{\hat{Q}} \approx$ const) or statistical fluctuations (i.e., $\sigma_{\hat{Q}} \propto\langle\hat{Q}\rangle^{1 / 2}$ ) [30].

When the beam is in a quasiequilibrium state, the onaxis charge (or equivalently $\hat{s}$ ) can be a single parameter that effectively characterizes the variation of the equilibrium density profile from one error sample to another [17]. Hence, except for the case where there is a significant departure from the equilibrium state (such as the formation of a broad halo), we can infer the statistical characteristics of the radial density profile from those of the on-axis charge. For example, when the normalized intensity parameter $\hat{s}$ is moderately low ( $\hat{s} \sim 0.2$ in this study), the equilibrium density profile of the beam is nearly Gaussian [18]. In this case, we can relate $N$ and $R_{b}$ to the on-axis density $\hat{n}=n(r=0)$ by $N \approx \hat{n} \pi R_{b}^{2}$. Since, $N$ is constant unless beam particles are lost to the wall, we can further consider $R_{b}$ to be a function of the parameter $\hat{n}$ according 

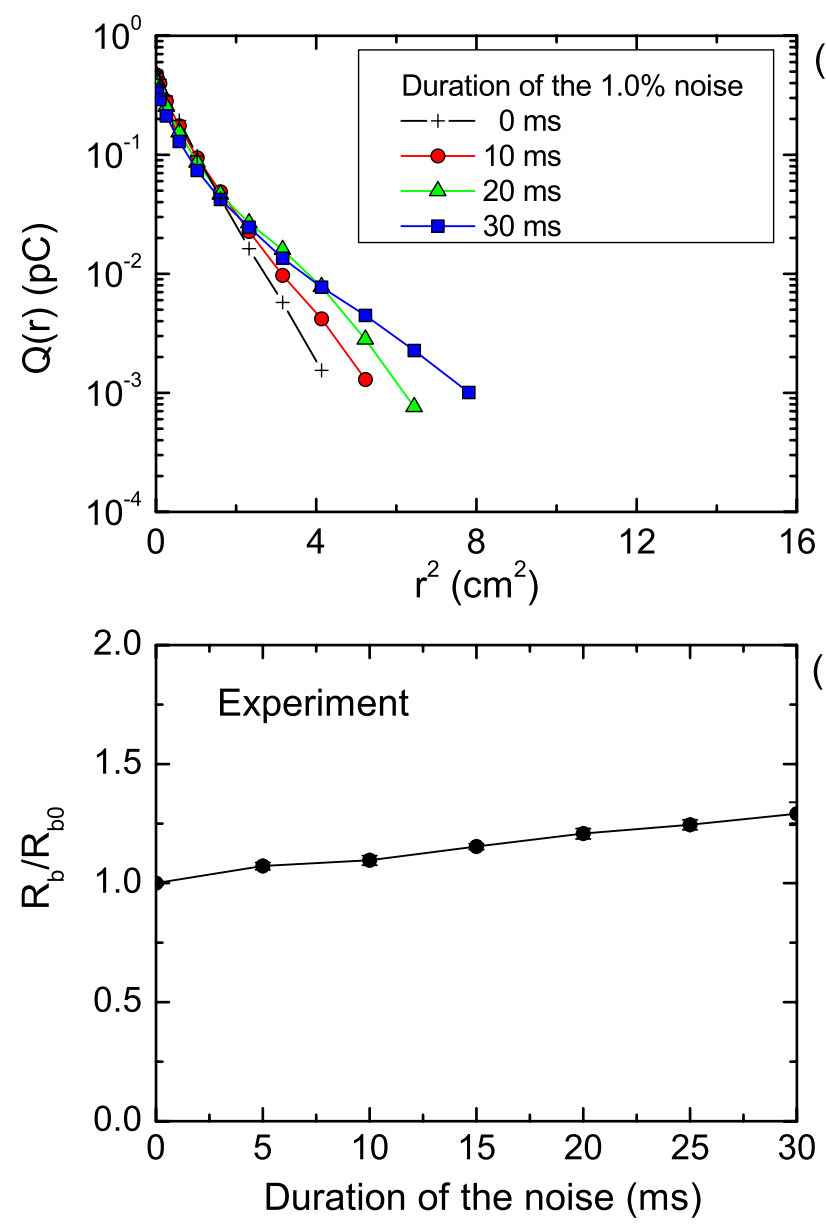

FIG. 5. (Color) Evolution of (a) the measured radial profile, and (b) the corresponding rms radius under the influence of $1 \%$ uniform white noise.

to $R_{b}(\hat{n}) \approx \sqrt{N / \pi \hat{n}}$. From Fig. 4, we can infer that the statistical fluctuations in $\hat{n}$ around its ensemble average value $\langle\hat{n}\rangle$ are small, i.e., $\hat{n}=\langle\hat{n}\rangle+\delta \hat{n}$ with $\langle\delta \hat{n}\rangle=0$ and $|\delta \hat{n} /\langle\hat{n}\rangle| \ll 1$. Therefore, for a given noise amplitude and duration, we obtain the following simple expressions for the ensemble average of the rms radius $\left\langle R_{b}\right\rangle$ and its variance $\sigma_{R_{b}}^{2}$ :

$$
\begin{gathered}
\left\langle R_{b}\right\rangle \approx \sqrt{\frac{N}{\pi\langle\hat{n}\rangle}}, \\
\sigma_{R_{b}}^{2} \approx \frac{N}{4 \pi} \frac{\sigma_{\hat{n}}^{2}}{\langle\hat{n}\rangle^{3}} .
\end{gathered}
$$

The error propagation formula [31] has been applied in obtaining Eq. (6). Although these approximate expressions are not explicitly used, they provide an indication that if there is a correlation between the on-axis density $\hat{n}$ and the rms radius $R_{b}$ (or equivalently emittance), then we can infer an rms radius averaged over the ensemble of error samples by using the statistical properties of the on-axis density. By measuring a single radial profile for a given noise amplitude and duration with a certain error sample that gives $\hat{n} \approx\langle\hat{n}\rangle$, and calculating the corresponding rms radius using $R_{b}^{2}=(1 / N) \int_{0}^{r_{w}} n(r) 2 \pi r^{3} d r$, we can effectively estimate $\left\langle R_{b}\right\rangle$. Otherwise, it would be necessary to measure $n(r)$ for every error sample to calculate the ensemble-averaged quantities, which requires 360 independent radial profile measurements to scan the entire parameter range presented in this study. This process would be very demanding for the present diagnostic setup, and is vulnerable to any drift in the experimental conditions (mostly from the ion source). In the present study, the processes of choosing an appropriate error sample and measuring the corresponding radial profile have been performed in $5 \mathrm{~ms}$ intervals (300 focusing periods) with three different noise amplitudes, which require only 18 independent radial profile measurements. In Figs. 5 and 7, the
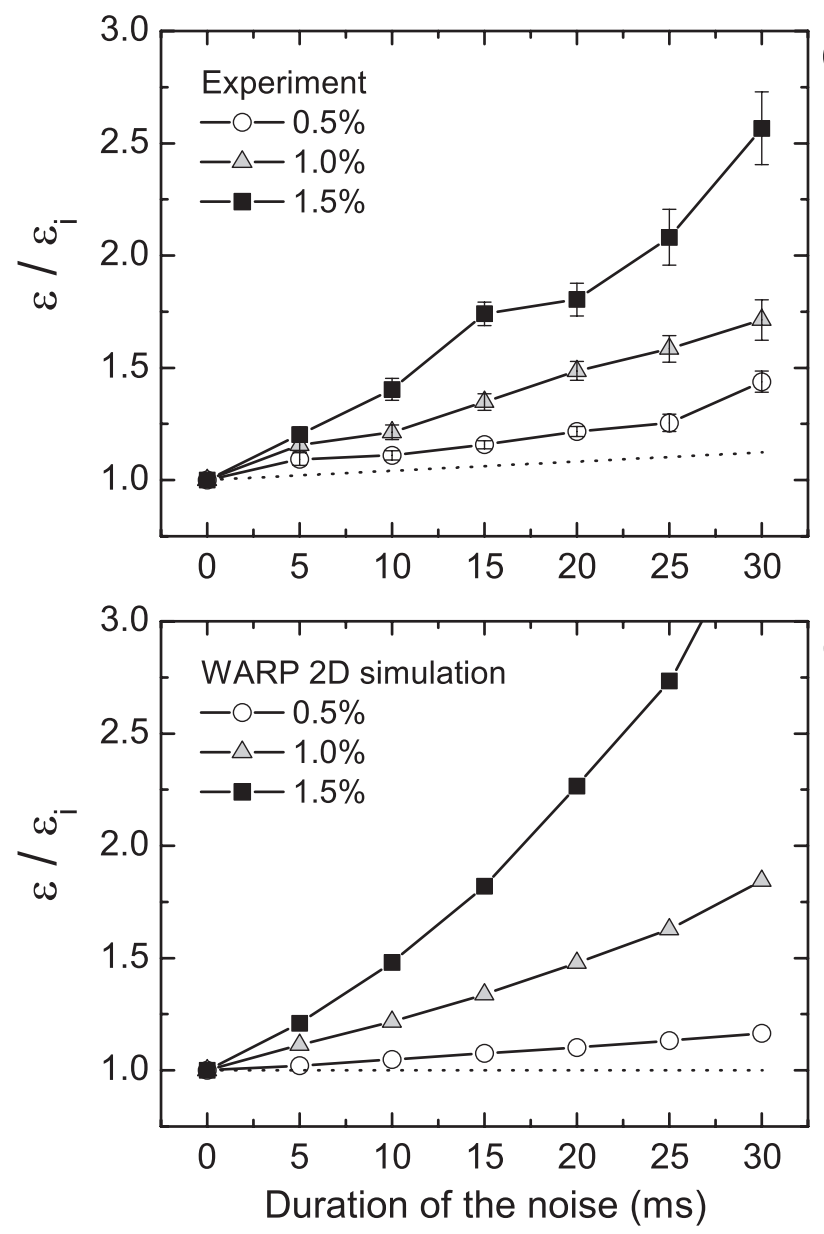

FIG. 6. The emittance growth is estimated from (a) radial profile measurements, and (b) WARP 2D PIC simulations. The emittance is calculated from Eq. (4), and is normalized to its initial value $\epsilon_{i}$. For the WARP simulations, 20 random error samples are used to calculate the ensemble-averaged emittance. The dotted lines indicate the background emittance growth in the absence of the applied noise. 


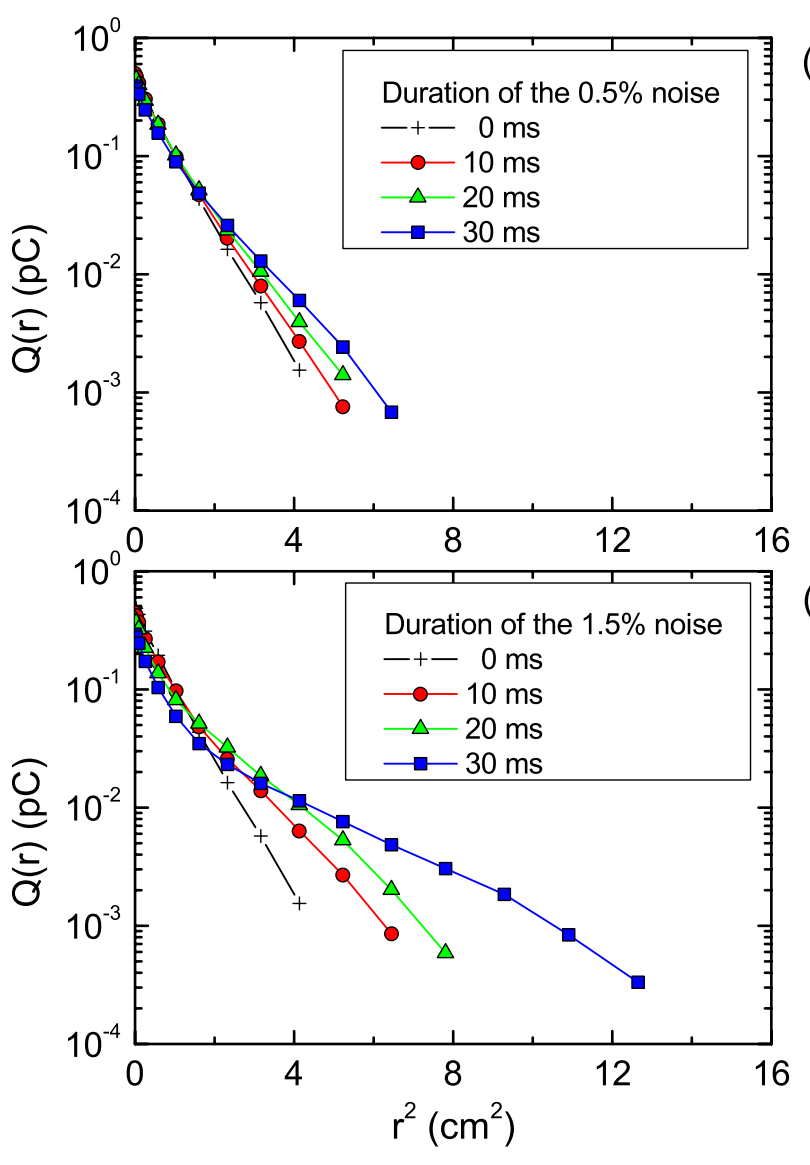

FIG. 7. (Color) Measured radial profiles with different noise amplitudes and duration. Initially, the trapped plasma is in a thermal equilibrium state, for which the radial density profile is a straight line in the $\log$ versus $r^{2}$ plot.

measured radial charge profiles are shown in $10 \mathrm{~ms}$ intervals rather than $5 \mathrm{~ms}$ intervals without error bars to indicate the evolution of the low-density tail more clearly. The typical scale of the error bars for these plots is similar to that of Fig. 3.

The typical evolution of the measured radial profiles under the influence of uniform white noise (with $\Delta_{\max }=$ 1\%) is shown in Fig. 5(a). It is clear that low-density tails are developing in the radial charge profiles, and the corresponding rms radius $R_{b}$ grows almost linearly with the noise duration [see Fig. 5(b)]. Note that there is good agreement between the rms radius obtained in the WARP 2D simulations presented in Fig. 2(b) and the experimental results presented in Fig. 5(b). The evolution of the average transverse emittance given in Eq. (4) is estimated from the radial profile measurements for a given noise amplitude and duration, using a total of 18 independent radial profiles. Consistent with the WARP 2D simulations, we observe a continuous emittance growth which is approximately linear with the duration of the noise (Fig. 6). We note from Figs. 2(b) and 5(b) that the rms radius $R_{b}$ increases approximately linearly with time as $R_{b} / R_{b 0} \sim$ $1+\gamma t$, where $\gamma \approx$ const. Hence, using Eq. (4), we would expect the emittance to increase according to $\epsilon / \epsilon_{i} \sim$ $\left(R_{b} / R_{b 0}\right)^{2} \sim(1+\gamma t)^{2} \sim 1+2 \gamma t$, for small growth rate $\gamma$. If the growth rate becomes sufficiently large, the emittance increases approximately parabolically with time. For the case where $\Delta_{\max }=1.5 \%$, the experimentally determined emittance is somewhat underestimated after a noise duration of $15 \mathrm{~ms}$. This is most likely due to the formation of a significant halo population under the detection limit ( $\sim 1 \mathrm{fC}$ ) of the charge collector, which is too low to be measured in the experiment, but contributes considerably in the simulations. The formation of a significant halo population is apparent in Fig. 7(b). On the other hand, for the case with $\Delta_{\max }=0.5 \%$, the experimentally estimated emittance has a slightly larger value than the simulation results, which is likely due to the intrinsic noise present in the PTSX device. Since the correlation between the intrinsic and applied noise, and its effect on the resultant emittance growth are somewhat unclear, the background emittance growth level is plotted separately, rather than with the standard procedure of subtraction. For the WARP 2D PIC simulation presented in Fig. 6(b), 40000 macroparticles are used, which is comparable to the number of macroparticles adopted for other noise simulations [8]. The time step for the PIC simulation is $\Delta t=$ $0.05 / f_{0} \ll 2 \pi / \omega_{p}$.

\section{EFFECTS OF COLORED NOISE}

So far, we have used uniform white noise to model random errors in the quadrupole focusing gradient. In an actual accelerator system, a power supply usually drives several magnets in series which are mounted on a common mechanical structure. Thus, any ripples in the driven currents or ground vibration can comprise a colored noise in the quadrupole focusing gradient with a finite autocorrelation time (e.g., several focusing periods) [32]. In recent papers $[4,7]$, Bohn and Sideris pointed out that the presence of colored noise can boost a small number of particles to much larger amplitudes than inferred from a parametric resonance alone [33], by continually kicking halo particles back into the right phase with the core envelope oscillation. Hence, in this section, we describe a preliminary experimental study that investigates the possible synergistic effect between colored noise and the collective modes indicated by Bohn and Sideris.

To modify the voltage amplitude with colored noise in every half-focusing period $T / 2$, we use a numerical algorithm generating Gaussian colored noise, based on the integration of the Langevin equation according to [29]

$$
\delta_{i+1}=\delta_{i} e^{-T /\left(2 \tau_{a c}\right)}+w_{i} \Delta_{\max }\left(1-e^{-T / \tau_{a c}}\right)^{1 / 2} .
$$

Here, $w_{i}$ is a Gaussian random number generated anew at each step $i$ with zero mean and unit variance, $\tau_{a c}$ is the autocorrelation time which measures the memory of ran- 

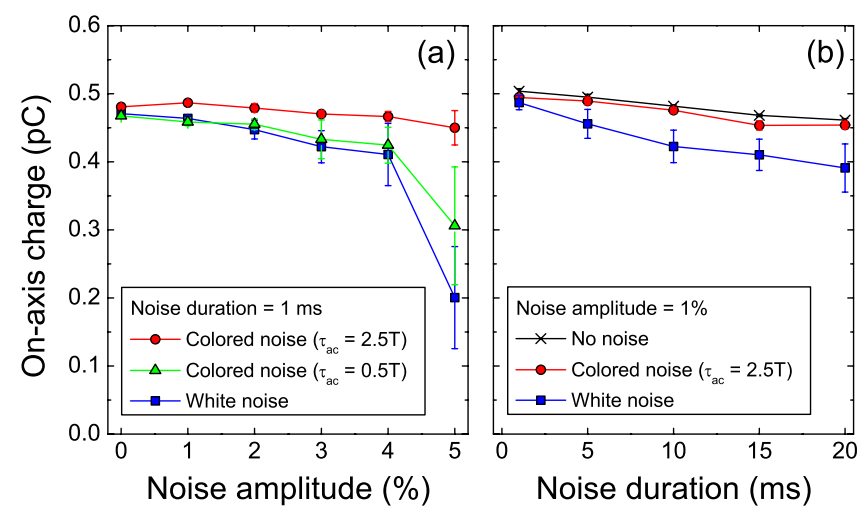

FIG. 8. (Color) Dependence of the on-axis charge signal on (a) noise amplitude, and (b) noise duration for white $\left(\tau_{a c}=0\right)$ and colored noise $\left(\tau_{a c}>0\right)$. The error bars indicate the standard deviation of the on-axis charge calculated over 20 error samples.

domness, and $\Delta_{\max }$ is the amplitude of the desired colored noise. To excite collective modes, we perturb the initial quiescent plasma $\left(\hat{s} \sim 0.2\right.$ and $\omega_{q}=52.2 \times 10^{3} \mathrm{~s}^{-1}$ for this case) by instantaneously increasing the voltage amplitude by 1.5 times, and switching back to the original value after one focusing period $T$ [34]. In this way, it is expected that a mixed mode with breathing mode period $2 \pi / \omega_{B} \approx$ $3.70 T$ and quadrupole mode period $2 \pi / \omega_{Q} \approx 3.65 T$ is excited [35]. Here, the breathing mode frequency $\omega_{B}$ and the quadrupole mode frequency $\omega_{Q}$ are expressed as [36]

$$
\begin{aligned}
& \omega_{B} \approx 2 \omega_{q}\left[1-\frac{1}{2}\left(\frac{K}{2 R_{b 0}^{2} \omega_{q}^{2}}\right)\right]^{1 / 2}, \\
& \omega_{Q} \approx 2 \omega_{q}\left[1-\frac{3}{4}\left(\frac{K}{2 R_{b 0}^{2} \omega_{q}^{2}}\right)\right]^{1 / 2},
\end{aligned}
$$

where $R_{b 0}$ is the equilibrium rms beam radius. Indeed, the breathing mode frequency is identical to the frequency of small-amplitude oscillations in the envelope equation (1). Note also that the two frequencies are both equal to $2 \omega_{q}$ in the absence of space-charge effect $(K \rightarrow 0)$.

For a matched beam, where there is no mismatch oscillation, scans of the noise amplitude and duration in Fig. 8 demonstrate that white noise is more detrimental than colored noise. Indeed, when the autocorrelation time of colored noise is greater than $2.5 T$, there is no significant change in the on-axis signal even for large amplitude and long duration of the colored noise. This can be understood because the energy kicks introduced by the white noise transfer maximum external energy into the system by compressing the beam most abruptly. As reported previously in Ref. [37], a gradual change in the focusing field strength tends to compress (or expand) the beam with less emittance growth. (a) Colored noise only

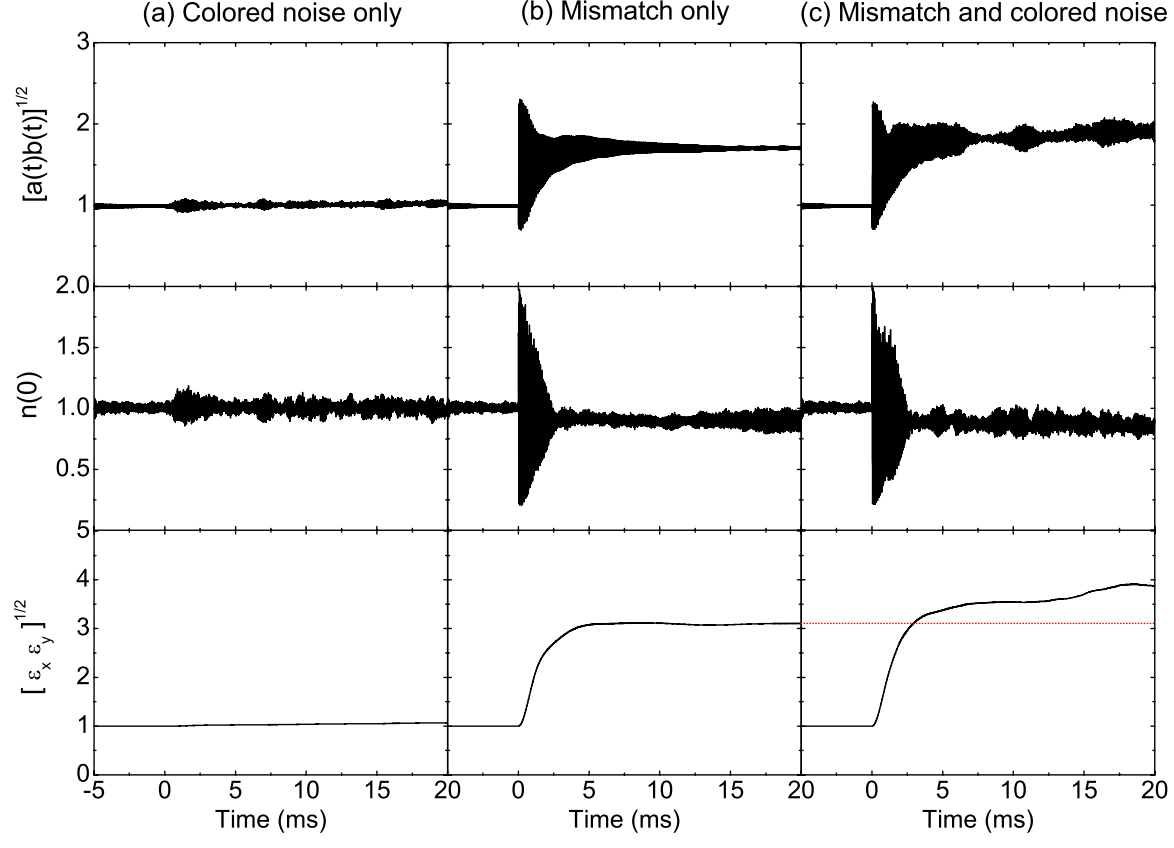

FIG. 9. WARP 2D PIC simulation results for the beam responses to (a) colored noise with $\tau_{a c}=5 T$ and $\Delta_{\text {max }}=1 \%$, (b) beam mismatch introduced by the incorrect (1.5 times stronger in this case) focusing field strength for one FODO lattice period, and (c) the combination of colored noise and beam mismatch. The mean radius $[a(t) b(t)]^{1 / 2}$ (top), the on-axis density $n(0)$ (middle), and the mean transverse emittance $\left[\epsilon_{x} \epsilon_{y}\right]^{1 / 2}$ (bottom) are normalized to their initial values, respectively, and perturbation starts at $t=0 \mathrm{~ms}$. For a fair comparison, the same colored noise sample has been applied to cases (a) and (c). 
The simulation results in Fig. 9(a) also indicate that the colored noise $\left(\tau_{a c}=5 T\right.$ for this case) perturbs the initial matched beam only slightly and leaves the average on-axis density nearly unchanged, as expected from the experimental results in Fig. 8. Here, we have chosen the autocorrelation time to be $\tau_{a c}=5 T$ so that the colored noise itself will not affect the initial beam too much (when $\tau_{a c}>$ $2.5 T$ ), and have a time scale comparable to the collective modes (for instance, $2 \pi / \omega_{B} \approx 3.70 T$ ). From the beam mismatch case presented in Fig. 9(b), we observe the excitation of collective modes, resulting in a $200 \%$ emittance growth. The envelope oscillations last for about $5 \mathrm{~ms}$ and eventually damp away. The most interesting case is the beam response in the presence of both the collective modes and the colored noise [Fig. 9(c)]. Even though the colored noise itself cannot excite significant envelope oscillations, when combined with the collective modes, it gives rise to continuous emittance growth and an increase in the mean radius with much higher oscillation amplitudes. These simulation results can also be interpreted as indicating

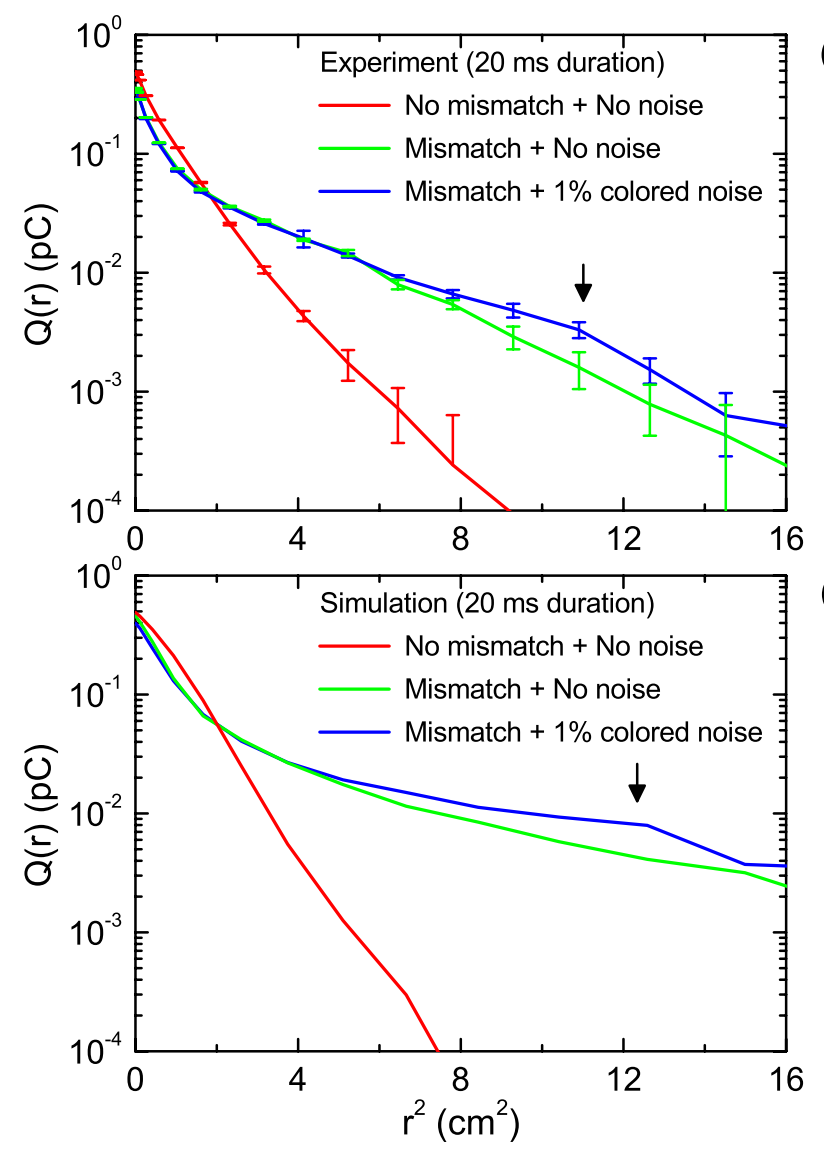

FIG. 10. (Color) Radial profiles are either (a) measured from the experiments, or (b) obtained from the PIC simulations. Three different external perturbation scenarios are considered: no perturbation (red curves); instantaneous mismatch only (green curves); and both instantaneous mismatch and colored noise with $\tau_{a c}=5 T$ and $\Delta_{\max }=1 \%$ (blue curves). enhanced halo formation. One possible underlying mechanism for enhanced halo formation has been proposed by Bohn and Sideris [4,7]. By extending the particle-core model $[33,38]$, they showed that the combination of colored noise and core envelope oscillations (breathing modes in their case) can eject particles to a much larger degree than would be achieved in the absence of noise.

To experimentally explore the synergistic effect between collective modes and colored noise, and the resultant enhanced halo formation predicted in the simulations, we measured the radial charge profiles corresponding to cases (b) and (c) of Fig. 9. In the case where there is neither induced mismatch nor applied noise, the initial matched beam remains nearly in a quasiequilibrium state (with $R_{b}=0.916 \mathrm{~cm}$ ) even after $20 \mathrm{~ms}$ of trapping [red curve in Fig. 10(a)]. On the other hand, when the beam is instantaneously mismatched in the same manner as in the previous simulation, a large nonthermal ion tail is measured at $r>\sqrt{2} R_{b} \approx 1.29 \mathrm{~cm}$ [green curve in Fig. 10(a)]. More interestingly, in the experiment with both instantaneous mismatch and applied colored noise, we observe the development of a small bump around $\approx(3 \sim 4) R_{b}$ [blue curve and arrow in Fig. 10(a)]. The error bars are determined primarily from the offset errors $(\sim 1 \mathrm{fC})$ in constructing the radial profiles. Although the accuracy is not adequate to cover the range below $\lesssim 1 \mathrm{fC}$, it still gives enough precision to resolve the small bump illustrated in Fig. 10(a). Therefore, the experimentally observed bump might be attributed to the effect of the enhanced halo formation expected for the case of combined perturbations. To check the size and the location of the bump, we also perform WARP 2D PIC simulations for similar experimental parameters. Despite the difference in their absolute values, the relative sizes and locations of the bumps illustrate very good agreement between the experimental results and the simulations [see Fig. 10(b)]. The discrepancy in the absolute value is likely related to 3D end effects present in the PTSX device, which could enhance the radial particle loss to the wall for particles with large radial excursion [26].

\section{SUMMARY AND DISCUSSION}

In this study, we have presented experimental verification of the random noise-induced beam degradation theoretically expected in high-intensity accelerators. This was possible because the PTSX device is a compact experimental setup with flexible control over the external focusing fields that can simulate the nonlinear transverse dynamics of an intense beam propagating through an actual AG focusing system. By adding a small random ripple on top of the applied voltage waveform, we demonstrated that externally driven noise continuously increases the rms radius, transverse emittance, and nonthermal tail of the trapped charge bunch depending on the amplitude and duration of the noise. In particular, we have observed the 
combined effects of collective modes and colored noise, which are consistent with theoretical predictions and numerical simulations.

The amplitude of the noise $\left(\Delta_{\max } \approx 1 \%\right)$ used to emulate machine imperfection effects in this study may seem somewhat larger than the actual tolerance limits adopted in daily accelerator operation. This is a practical compromise to overcome the difficulty of measuring small changes in charge signals $(\sim 1 \mathrm{fC})$ in the PTSX device (particularly for the tail distribution or halo particles in the off-axis regions). Nonetheless, in modern high-intensity accelerators, loss of only a few particles per meter can cause radioactivation that would preclude routine hands-on maintenance [4]. Therefore, it is highly relevant to verify the validity of numerical tools and to test the physics models for beam loss in experiments with parameters even somewhat beyond the actual tolerance limits. For the present study, the combined effect of collective modes and colored noise has been investigated with a fixed autocorrelation time $\tau_{a c}=5 T$. By measuring radial profiles with several different autocorrelation times, e.g., $2 \pi / \tau_{a c} \approx \omega_{q}, \omega_{B}, \omega_{Q},\left(\omega_{Q}+\omega_{B}\right) / 2,\left(\omega_{Q}-\omega_{B}\right) / 2, \ldots$, it may be possible to develop a more detailed understanding of noise-enhanced halo formation (for example, how the location of the bump in the radial profile changes). Moreover, future experiments with different (either very large or very small) space-charge contribution are expected to further improve our basic understanding of the role of nonlinear space-charge force in random noise-induced beam degradation. Improving the accuracy of the charge collector diagnostic will also facilitate a more detailed resolution of the small changes expected in this type of experiment.

\section{ACKNOWLEDGMENTS}

This research was supported by the U.S. Department of Energy. The authors would like to thank Andy Carpe for his excellent technical support, and Mikhail Dorf for useful discussions regarding the WARP simulations. The research was carried out at Plasma Physics Laboratory while the corresponding author (Moses Chung) was at Princeton University.

[1] R. C. Davidson and H. Qin, Physics of Intense Charged Particle Beams in High Energy Accelerators (World Scientific, Singapore, 2001), Chap. 1, and references therein.

[2] M. Reiser, Theory and Design of Charged Particle Beams (Wiley-VCH, Weinheim, 2008), 2nd ed., Chap. 7.

[3] T. Wangler, RF Linear Accelerators (Wiley-VCH, Weinheim, 2008), 2nd ed., Chap. 12.

[4] C. L. Bohn and I. V. Sideris, Phys. Rev. Lett. 91, 264801 (2003).

[5] F. Gerigk, Phys. Rev. ST Accel. Beams 7, 064202 (2004).
[6] J. Qiang, R. D. Ryne, B. Blind, J.H. Billen, T. Bhatia, R. W. Garnett, G. Neuschaefer, and H. Takeda, Nucl. Instrum. Methods Phys. Res., Sect. A 457, 1 (2001).

[7] I. V. Sideris and C. L. Bohn, Phys. Rev. ST Accel. Beams 7, 104202 (2004).

[8] G. Franchetti and I. Hofmann, in Proceedings of 2002 European Particle Accelerator Conference, Paris, France (EPS-IGA/CERN, Geneva, 2002), p. 1353.

[9] F. Gerigk, in Beam Halo Dynamics, Diagnostics, and Collimation: 29th ICFA Advanced Beam Dynamics Workshop on Beam Halo Dynamics, Diagnostics, and Collimation, AIP Conf. Proc. No. 693 (American Institute of Physics, New York, 2003), p. 61.

[10] D. V. Gorelov and P. N. Ostroumov, in Proceedings of the 1998 International Linear Accelerator Conference, Chicago (ANL, Argonne, 1998), p. 654.

[11] M. Ikegami, T. Ohkawa, Y. Kondo, and A. Ueno, in Proceedings of 2004 International Linear Accelerator Conference, Lubeck, Germany (DESY/GSI, Darmstadt, Germany, 2004), p. 345.

[12] P. S. Yoon, W. Chou, and C. L. Bohn, in Proceedings of the 2005 Particle Accelerator Conference, Knoxville, Tennessee (IEEE, Piscataway, NJ, 2005), p. 117.

[13] W. Paul and H. Steinwedel, Z. Naturforsch. A 8, 448 (1953).

[14] R. C. Davidson, H. Qin, and G. Shvets, Phys. Plasmas 7, 1020 (2000).

[15] E. P. Gilson, R.C. Davidson, P.C. Efthimion, and R. Majeski, Phys. Rev. Lett. 92, 155002 (2004).

[16] H. Okamoto and H. Tanaka, Nucl. Instrum. Methods Phys. Res., Sect. A 437, 178 (1999).

[17] R. C. Davidson and H. Qin, Phys. Rev. ST Accel. Beams 2, 114401 (1999).

[18] See, for example, Chaps. 3 and 5 of Ref. [1].

[19] G. Franchetti, I. Hofmann, and D. Jeon, Phys. Rev. Lett. 88, 254802 (2002).

[20] S. Henderson, I. Anderson, N. Holtkamp, and C. Strawbridge, SNS Parameters List (SNS-100000000PL0001-R13) (Oak Ridge National Laboratory, Oak Ridge, TN, 2005).

[21] D. Jeon, SNS Superconducting Linac Beam Dynamics (SNS/ORNL/AP Technical Note 12) (Oak Ridge National Laboratory, Oak Ridge, TN, 1999).

[22] M. Chung, E. P. Gilson, M. Dorf, R. C. Davidson, P. C. Efthimion, and R. Majeski, Phys. Rev. ST Accel. Beams 10, 014202 (2007).

[23] A. V. Fedotov, Nucl. Instrum. Methods Phys. Res., Sect. A 557, 216 (2006).

[24] E. P. Gilson, R. C. Davidson, P. C. Efthimion, R. Majeski, and H. Qin, Laser Part. Beams 21, 549 (2003).

[25] E. P. Gilson, M. Chung, R.C. Davidson, P.C. Efthimion, R. Majeski, and E. A. Startsev, Nucl. Instrum. Methods Phys. Res., Sect. A 544, 171 (2005).

[26] M. Chung, Ph.D. thesis, Princeton University, Princeton, NJ, 2008.

[27] A. Friedman, D. P. Grote, and I. Haber, Phys. Fluids B 4, 2203 (1992).

[28] M. Reiser, J. Appl. Phys. 70, 1919 (1991).

[29] T. Stoltzfus-Dueck and J. A. Krommes (private communication).

[30] P. R. Bevington and D. K. Robinson, Data Reduction and 
Error Analysis for the Physical Sciences (McGraw-Hill, New York, 2003), 3rd ed.

[31] Using Eq. (3.14) of Ref. [30], we find $\sigma_{R_{b}}^{2} \approx$ $\sigma_{\hat{n}}^{2}\left(\partial R_{b} / \partial \hat{n}\right)_{\hat{n}=\langle\hat{n}\rangle}^{2} \approx \sigma_{\hat{n}}^{2}(N / 4 \pi)\langle\hat{n}\rangle^{-3}$.

[32] S.Y. Lee, Accelerator Physics (World Scientific, Singapore, 2004), Chap. 2.

[33] R. L. Gluckstern, Phys. Rev. Lett. 73, 1247 (1994).

[34] E. P. Gilson, M. Chung, R. C. Davidson, M. Dorf, P. C. Efthimion, and R. Majeski, Phys. Plasmas 13, 056705 (2006).
[35] See, for example, Chaps. 4 of Ref. [2].

[36] E. P. Gilson, M. Chung, R. C. Davidson, M. Dorf, P. C. Efthimion, A. B. Godbehere, and R. Majeski, Nucl. Instrum. Methods Phys. Res., Sect. A (to be published).

[37] M. Chung, E. P. Gilson, M. Dorf, R. C. Davidson, P. C. Efthimion, and R. Majeski, Phys. Rev. ST Accel. Beams 10, 064202 (2007).

[38] T. P. Wangler, K. R. Crandall, R. Ryne, and T. S. Wang, Phys. Rev. ST Accel. Beams 1, 084201 (1998). 